\title{
多级孔 $\mathrm{Co} / \mathrm{Al}-\mathrm{SiO}_{2}$ 催化剂制备及其费-托合成催化性能
}

\author{
王举汉，文雄，刘成超，张煜华，赵燕喜，李金林 \\ (中南民族大学 催化转化与能源材料化学教育部重点实验室, 催化材料科学湖北省重点实验室, 武汉 430074)
}

摘 要: 多级孔硅铝材料因优异的性能而成为金属催化剂的重要载体。研究采用水热法以硝酸铝为铝源、正硅酸乙 酯(TEOS)为硅源合成了 $\mathrm{Al}$ 掺杂 $\mathrm{SiO}_{2}\left(\mathrm{Al}_{-}-\mathrm{SiO}_{2}\right)$ 的多级孔材料, 然后采用等体积浸渍法负载钴, 制备了具有多级孔结 构的 $\mathrm{Co} / \mathrm{Al}-\mathrm{SiO}_{2}$ 催化剂。对 $\mathrm{Al}_{-} \mathrm{SiO}_{2}$ 材料的表征结果表明: 以四丙基氢氧化铵(TPAOH)为结构导向剂, 经 $80{ }^{\circ} \mathrm{C}$ 水 热处理可以合成多级孔 $\mathrm{Al}^{-} \mathrm{SiO}_{2}$, 呈尺寸在 30 40 nm 范围的 “蠕虫状”大孔分布形态。将催化剂用于费-托合成反应, 与商业 $\mathrm{SiO}_{2}$ 负载的钴催化剂相比, $\mathrm{Co} / \mathrm{Al}-\mathrm{SiO}_{2}$ 的 $\mathrm{CO}$ 转化率提高近一倍, $\mathrm{CH}_{4}$ 选择性降低 $19.3 \mathrm{wt} \%, \mathrm{C}_{2}-\mathrm{C}_{4}$ 选择性降低 $13.3 \mathrm{wt} \%$, 汽油段产物 $\left(\mathrm{C}_{5}-\mathrm{C}_{12}\right)$ 选择性达到 $53.3 \mathrm{wt} \%$ 。

关 键 词: 水热法; 多级孔材料; $\mathrm{Al}-\mathrm{SiO}_{2}$; 钴催化剂; 费-托合成

中图分类号: O643 文献标识码: A

\section{Preparation and Fischer-Tropsch Synthesis Performance of Hierarchical Co/Al-SiO 2 Catalyst}

WANG Juhan, WEN Xiong, LIU Chengchao, ZHANG Yuhua, ZHAO Yanxi, LI Jinlin

(Hubei Key Laboratory of Catalysis and Materials Science, Key Laboratory of Catalysis and Energy Materials Chemistry of Ministry of Education, South-Central University for Nationalities, Wuhan 430074, China)

\begin{abstract}
Hierarchically porous silica-aluminum is an important support material for metal-catalysts, because of its excellent properties. Herein, an efficient hydrothermal approach for the production of hierarchical aluminumdoped silica $\left(\mathrm{Al}-\mathrm{SiO}_{2}\right)$ architectures was reported by employing aluminum nitrate as an aluminium source and TEOS as silicon source. Effects of the structure-oriented agent on the structure of $\mathrm{Al}-\mathrm{SiO}_{2}$ were investigated. Structural features of $\mathrm{Al}_{-} \mathrm{SiO}_{2}$ were characterized by XRD, SEM and $\mathrm{N}_{2}$-physisorption. The results showed that hierarchical $\mathrm{Al}-\mathrm{SiO}_{2}$ with "worm-like" porous of $30-40 \mathrm{~nm}$ can be synthesized by using TPAOH as the structure-oriented agent and hydrothermal treatment at $80{ }^{\circ} \mathrm{C}$. The cobalt catalysts were prepared by the wetness impregnation method. Compared with commercial $\mathrm{SiO}_{2}$ supported cobalt catalysts, the Fischer-Tropsch synthesis performance of the catalyst $\mathrm{Co} / \mathrm{Al}-\mathrm{SiO}_{2}$ is significantly enhanced, i.e., $\mathrm{CO}$ conversion nearly doubled, $\mathrm{CH}_{4}$ selectivity reduced by $19.3 \mathrm{wt} \%, \mathrm{C}_{2}-\mathrm{C}_{4}$ selectivity reduced by $13.3 \mathrm{wt} \%$, and the selectivity of gasoline products $\left(\mathrm{C}_{5}-\mathrm{C}_{12}\right)$ reached $53.3 \mathrm{wt} \%$.
\end{abstract}

Key words: hydrothermal method; hierarchical porous materials; $\mathrm{Al}_{-} \mathrm{SiO}_{2}$; Cobalt catalyst; Fischer-Tropsch synthesis

费托合成(FTS)是将来源于煤、天然气和生物质

等碳资源的合成气 $\left(\mathrm{CO}+\mathrm{H}_{2}\right)$ 转化为清洁液体燃料和

收稿日期: 2019-09-29; 收到修改稿日期：2019-12-17

基金项目：国家自然科学基金(21972170, 21902187); 湖北省自然科学基金(2018CFB365)

National Natural Science Foundation of China (21972170, 21902187); Natural Science Foundation of Hubei Province of China (2018CFB365)

作者简介: 王举汉(1994-), 男, 硕士研究生. E-mail: 834767117@qq.com

WANG Juhan(1994-), male, master candidate. E-mail: 834767117@qq.com

通讯作者: 李金林，教授.E-mail: jinlinli@aliyun.com

LI Jinlin, professor. E-mail: jinlinli@aliyun.com 
化学品的一种重要方法 ${ }^{[1-4]}$ 。铁和钴催化剂是费托合 成常用的催化剂, 与铁催化剂相比, 钴催化剂具有 较高的活性、较高 $\mathrm{C}_{5+}$ 的选择性和较低的水煤气反 应活性等特点。

介孔 $\mathrm{SiO}_{2}$ 是一种常用的费托合成钴催化剂载 体 ${ }^{[5-10]}$ 。Song 等 ${ }^{[11]}$ 研究了 $\mathrm{SiO}_{2}$ 载体介孔孔径对负载 的钴催化剂费-托合成性能的影响, 发现载体孔径 影响催化剂上钴物种的分散度和还原性, 进而影响 催化剂的活性和选择性, 当载体孔径为 $6 \sim 10 \mathrm{~nm}$ 时, 催化剂具有最优的费-托合成活性和 $\mathrm{C}_{5+}$ 选择性。 Xiong 等 ${ }^{[6,12]}$ 研究了具有二维孔结构的 SBA-15 分子 篮的孔径对钴催化剂的费-托合成性能的影响, 发 现 $\mathrm{CO}$ 转化率随载体孔径增大先增加后降低, 当平 均孔径为 $6 \sim 10 \mathrm{~nm}$ 时 SBA-15 上负载的钴催化剂具 有最高的活性和重质烃选择性。

在介孔 $\mathrm{SiO}_{2}$ 负载的钴催化剂中引入大孔结构, 有利于反应物和产物分子的传质与扩散, 可提高催 化剂性能。Koo 等 ${ }^{[5]}$ 采用双模板法合成了介孔-大孔 $\mathrm{SiO}_{2}(\mathrm{MMS})$ 载体, 并制备了 $\mathrm{Co} / \mathrm{MMS}$ 催化剂, 费-托 合成催化性能测试结果显示引入大孔提高了催化剂 活性和重质烃产物选择性。相较于传统的介孔-大孔 $\mathrm{SiO}_{2}$ 载体, 三维多级孔泡沫硅材料(MCF)不仅比表 面积更高, 而且其三维互通大孔结构的传质效率更 高。最近, Wei 等 ${ }^{[9]}$ 研究了 MCF 负载钴催化剂的费托合成催化性能，相比 KIT-6、SBA-15、SBA-16 等 分子篮, $\mathrm{MCF}$ 负载的钴催化剂具有更高的活性和重 质烃选择性, 他们将该催化剂的良好性能归因于 $\mathrm{Co} / \mathrm{MCF}$ 催化剂具有大且开放互通的孔结构, 有利 于反应物的运输和二次反应的发生, 使催化剂具有 较高的活性和 $\mathrm{C}_{5}$ 选择性。虽然具有高比表面积和 大孔结构的 MCF 材料负载钴催化剂可以改善催化 剂的费-托合成催化性能。但是费托合成条件下钴物 种在大孔中易于迁移团聚，使催化剂的稳定性有所 欠缺, 加上较高的材料合成成本限制了 MCF 材料 的实际应用。

本研究通过调变结构导向剂和水热温度合成了 $\mathrm{Al}$ 掺杂 $\mathrm{SiO}_{2}$ 的多级孔 $\left(\mathrm{Al}-\mathrm{SiO}_{2}\right)$ 材料, 采用等体积浸 渍法制备了负载型钴催化剂, 以商业 $\mathrm{SiO}_{2}$ 为参比, 研究了多级孔 $\mathrm{Al}-\mathrm{SiO}_{2}$ 载体负载钴催化剂的费-托合 成催化性能。

\section{1 实验方法}

\section{1 原料}

四丙基氢氧化铵(四丙基氢氧化铵, $2.0 \mathrm{~mol} / \mathrm{L}\left(\mathrm{H}_{2} \mathrm{O}\right)$ ), 正硅酸四乙酯(TEOS, 纯度为 $98 \%$ ), 由阿拉丁公司
提供; 乙二醇胺 $\left(\mathrm{C}_{4} \mathrm{H}_{11} \mathrm{NO}_{2}\right.$, 化学纯), 苄胺 $\left(\mathrm{C}_{7} \mathrm{H}_{9} \mathrm{~N}\right.$, 化学 纯), 乙二胺 $\left(\mathrm{C}_{2} \mathrm{H}_{8} \mathrm{~N}_{2}\right.$, 化学纯), 硝酸铝 $\left(\mathrm{Al}\left(\mathrm{NO}_{3}\right)_{3} \cdot 9 \mathrm{H}_{2} \mathrm{O}\right.$, 分析纯), 硝酸钴 $\left(\mathrm{Co}\left(\mathrm{NO}_{3}\right)_{2} \cdot 6 \mathrm{H}_{2} \mathrm{O}\right.$, 分析纯 $)$, 由国药 集团化学试剂有限公司提供; 商业 $\mathrm{SiO}_{2}$ (青岛美高, 比表面积为 $297 \mathrm{~m}^{2} / \mathrm{g}$, 孔体积为 $1.0 \mathrm{~cm}^{3} / \mathrm{g}$, 平均孔 径为 $8.6 \mathrm{~nm}$ )。

\section{$1.2 \mathrm{Al}_{-} \mathrm{SiO}_{2}$ 材料及催化剂的制备}

\subsection{1 多级孔 $\mathrm{Al}^{-\mathrm{SiO}_{2}}$ 的制备}

在 $108 \mathrm{~mL} \mathrm{H} 2 \mathrm{O}$ 中, 加入 $1.2656 \mathrm{~g} \mathrm{Al}\left(\mathrm{NO}_{3}\right)_{3} \cdot 9 \mathrm{H}_{2} \mathrm{O}$ 搅拌至溶解, 然后向溶液中加入 $17 \mathrm{~mL}$ 四丙基氢氧 化铵(TPAOH)结构导向剂, 随后将 $41.6 \mathrm{~g}$ TEOS 缓 慢滴入溶液中, 在 $60{ }^{\circ} \mathrm{C}$ 油浴中反应 $12 \mathrm{~h}$ 。反应结束 后, 将前驱体溶液转移至聚四氟乙烯内衬的不锈钢 高压反应釜中，在 $80{ }^{\circ} \mathrm{C}$ 保温 $24 \mathrm{~h}$, 然后自然降至室 温。所得溶胶经离心、过滤和无水乙醇洗涤, 自然 烘干, 所得产物放入马弗炉中 $550{ }^{\circ} \mathrm{C}$ 焙烧 $5 \mathrm{~h}$ (升温 速率为 $2{ }^{\circ} \mathrm{C} / \mathrm{min}$ ) 得到多级孔 $\mathrm{Al}-\mathrm{SiO}_{2}$ 材料, 命名为 $\mathrm{Al}-\mathrm{SiO}_{2}(\mathrm{t}-80)$ 。

保持 $80{ }^{\circ} \mathrm{C}$ 水热温度不变, 调变结构导向剂为 二乙醇胺、乙二胺、茮胺制得的多级孔 $\mathrm{Al}-\mathrm{SiO}_{2}$ 材 料分别命名为: $\mathrm{Al}-\mathrm{SiO}_{2}(\mathrm{~d}) 、 \mathrm{Al}-\mathrm{SiO}_{2}(\mathrm{e}) 、 \mathrm{Al}-\mathrm{SiO}_{2}(\mathrm{~b})$ 。

以 $\mathrm{TPAOH}$ 为结构导向剂, 改变水热温度为 $100 、 120 、 140{ }^{\circ} \mathrm{C}$, 制得的多级 $\mathrm{Al}-\mathrm{SiO}_{2}$ 材料分别命 名为: $\mathrm{Al}-\mathrm{SiO}_{2}(100) 、 \mathrm{Al}-\mathrm{SiO}_{2}(120) 、 \mathrm{Al}-\mathrm{SiO}_{2}(140)$

\subsection{2 催化剂的制备}

分别以商业和 $\mathrm{Al}-\mathrm{SiO}_{2}(\mathrm{t}-80)$ 为载体, 以 $\mathrm{Co}\left(\mathrm{NO}_{3}\right)_{2} \cdot 6 \mathrm{H}_{2} \mathrm{O}$ 为钴源, 去离子水为浸渍液, 采用 等体积浸渍法制备钴负载量为 $15 \mathrm{wt} \%$ 的钴催化剂, 催化剂经 $100{ }^{\circ} \mathrm{C}$ 干燥和 $350{ }^{\circ} \mathrm{C}$ 焙烧, 分别命名为 $\mathrm{Co} / \mathrm{SiO}_{2}$ 和 $\mathrm{Co} / \mathrm{Al}-\mathrm{SiO}_{2}(\mathrm{t}-80)$ 。

\section{$1.3 \mathrm{Al}_{-} \mathrm{SiO}_{2}$ 材料及催化剂的表征}

采用扫描电子显微镜(Hitachi-48005, Hitachi)观 察 $\mathrm{Al}-\mathrm{SiO}_{2}$ 的形貌, 采用物理化学吸附仪(Autosorb$1-\mathrm{C}$ )表征 $\mathrm{Al}-\mathrm{SiO}_{2}$ 的孔结构特性, 采用 X 射线衍射仪 (Brucker Advance D8, Brucker)表征 $\mathrm{Al}-\mathrm{SiO}_{2}$ 及催化 剂的晶型结构, 利用透射电子显微镜(Tecnai $G^{2} 20$, FEI)表征催化剂的形貌和分散情况, 用催化剂多功 能表征仪(AMI-200, Zeton Altamira)表征催化剂的还 原性, 采用电感耦合等离子发射光谱仪测量(Optima 4300DV, PE)催化剂活性金属的实际含量。

\section{4 催化剂的费-托合成催化性能评价}

按照参考文献[3]对催化剂的费-托合成性能进 行评价: 将催化剂(约 $0.5 \mathrm{~g}$ )与 $5 \mathrm{~g}$ 石英砂混合, 放入 底部垫有石英棉的反应管中。在常压和高纯氢气氛 $\left(3 \mathrm{~L} /(\mathrm{h} \cdot \mathrm{g})\left(20{ }^{\circ} \mathrm{C}\right), 450{ }^{\circ} \mathrm{C}\right.$, 常压)中还原催化剂, 反 
应器的温度以 $2{ }^{\circ} \mathrm{C} / \mathrm{min}$ 升温速率从室温升至 $450{ }^{\circ} \mathrm{C}$, 还原 $10 \mathrm{~h}$ 后, 将反应器冷却到 $100{ }^{\circ} \mathrm{C}$ 。切换氢气为 合成气 $\left(\mathrm{H}_{2} / \mathrm{CO}=2\right.$, 空速为 $\left.4 \mathrm{~L} /(\mathrm{h} \cdot \mathrm{g})\left(>20^{\circ} \mathrm{C}\right)\right)$, 反应 管内的压力升至 $1.0 \mathrm{MPa}$ ，充分混合一段时间后，程 序升温至 $210{ }^{\circ} \mathrm{C}$ 开始反应。热阱 $\left(100{ }^{\circ} \mathrm{C}\right)$ 用于收集 反应产物中的蜡样和水样, 冷阱 $\left(-2{ }^{\circ} \mathrm{C}\right)$ 则用于收集 油样和水样。采用配备有 TCD 检测器的 Agilent MicroGC 3000A 型气相色谱仪对气态尾气进行在线 分析。采用 Agilent GC 6890N 型色谱离线分析样。 蜡样溶解于二硫化碳中, 用 Agilent GC 7890A 型色 谱进行离线分析。在反应平稳 $40 \mathrm{~h}$ 后接近稳定状态 时获得活性和产物选择性数据, 用总碳质量平衡法 ${ }^{[1]}$ 计算烃的选择性。

\section{2 结果与讨论}

\section{1 结构导向剂的影响}

采用不同结构导向剂合成样品的 SEM 照片如 图 1(A)所示, 以四丙基氢氧化铵为结构导向剂合成 的样品 $\mathrm{Al}-\mathrm{SiO}_{2}(\mathrm{t}-80)$ 具有丰富的相互连通的“蠕虫 状”均一孔结构，其它三种结构导向剂制备的 $\mathrm{Al}-\mathrm{SiO}_{2}$ 样品的孔是由 15 95 nm 大小不均一的 $\mathrm{SiO}_{2}$ 颗粒堆积而成, 孔结构均是由堆积孔组成, 无序且 不均一。样品的 XRD 图谱(图 1(B))表明: 四个样品 在 $2 \theta=18^{\circ} \sim 27^{\circ}$ 范围内均存在宽泛的衍射峰, 归属于 无定型二氧化硅; 没有发现 $\mathrm{Al}_{2} \mathrm{O}_{3}$ 的峰, 说明 $\mathrm{Al}$ 元 素均匀地分散在 $\mathrm{SiO}_{2}$ 表面或进入了 $\mathrm{SiO}_{2}$ 骨架中。 综上, 四丙基氢氧化铵作为结构导向剂可得到形貌 较好的无定型多级孔(介孔-大孔) $\mathrm{Al}-\mathrm{SiO}_{2}$ 材料。

\section{2 水热温度的影响}

样品 $\mathrm{Al}-\mathrm{SiO}_{2}(\mathrm{t}-80) 、 \mathrm{Al}-\mathrm{SiO}_{2}(100) 、 \mathrm{Al}-\mathrm{SiO}_{2}(120)$ 、 $\mathrm{Al}-\mathrm{SiO}_{2}(140)$ 的 SEM 表征结果如图 2(A)所示, 随着 水热处理温度升高, 无定型“蠕虫状”孔开始变得密 集, 形貌较为均一，但并没有完整的晶体颗粒，直 到 $140{ }^{\circ} \mathrm{C}$ 出现“砖块”晶体。从图 2(B)可以看出, 水 热温度在 $80 、 100 、 120{ }^{\circ} \mathrm{C}$ 时, 样品在 $2 \theta=18^{\circ} \sim 27^{\circ}$ 处 出现无定型 $\mathrm{SiO}_{2}$ 的衍射峰, 而水热温度在 $140{ }^{\circ} \mathrm{C}$ 时, 图谱呈现出典型的 ZSM-5 骨架结构特征衍射峰 ${ }^{[13-15]}$, 表明在较低的水热温度下, 游离态的 $\mathrm{Si}$ 物种和 $\mathrm{Al}$ 物种以无定型态结合在一起; 在较高的水热温度下, 结构导向剂诱导游离的 $\mathrm{Si}$ 和 $\mathrm{Al}$ 物种成核结晶, 开 始形成高结晶度的微孔 ZSM-5 晶体颗粒。

为了了解水热温度对 $\mathrm{Al}-\mathrm{SiO}_{2}$ 材料孔结构的影 响, 对材料进行了 $\mathrm{N}_{2}$ 物理吸附表征, 结果如图 3 和 表 1 所示。经过 $80 、 100$ 和 $120{ }^{\circ} \mathrm{C}$ 水热处理的样品 呈现出典型的第IV 类型吸附等温曲线, 并在相对压
(A)
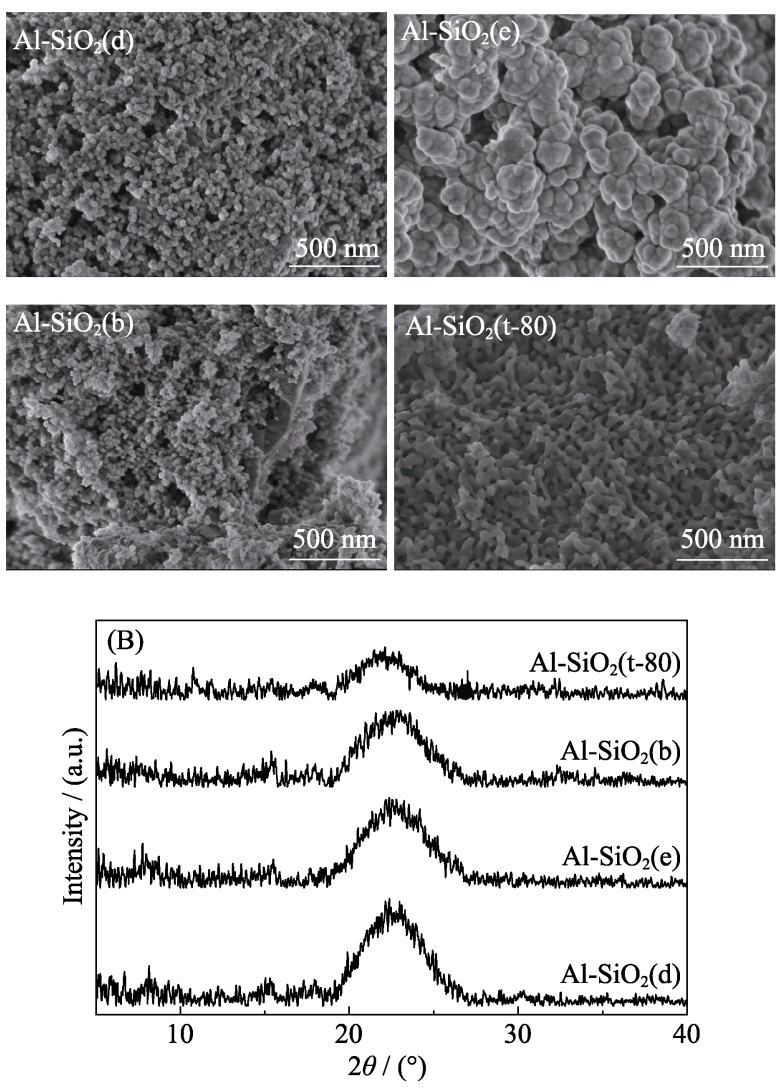

图 $1 \mathrm{Al}-\mathrm{SiO}_{2}$ 样品的 $\mathrm{SEM}$ 照片(A)和 XRD 图谱(B)

Fig. 1 SEM images (A) and XRD patterns(B) of $\mathrm{Al}-\mathrm{SiO}_{2}$
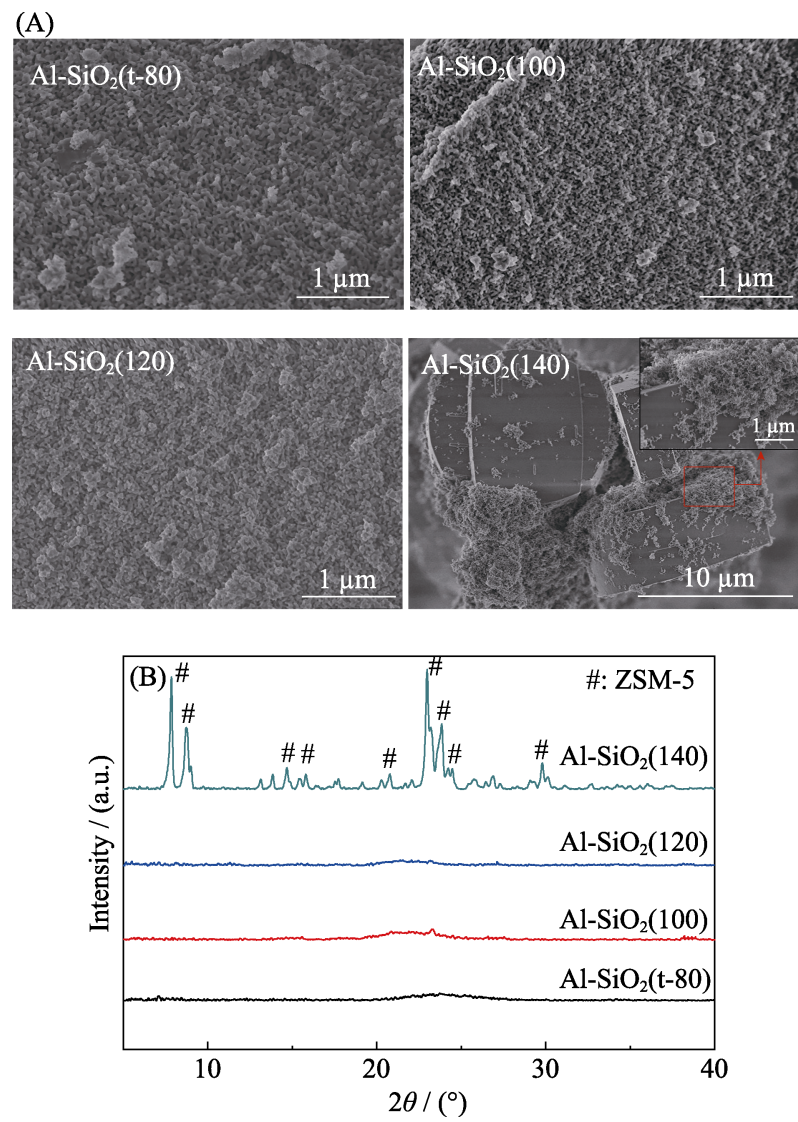

图 $2 \mathrm{Al}-\mathrm{SiO}_{2}$ 样品的 $\mathrm{SEM}$ 照片(A)和 XRD 图谱(B)

Fig. 2 SEM images (A) and XRD patterns (B) of $\mathrm{Al}-\mathrm{SiO}_{2}$ samples 

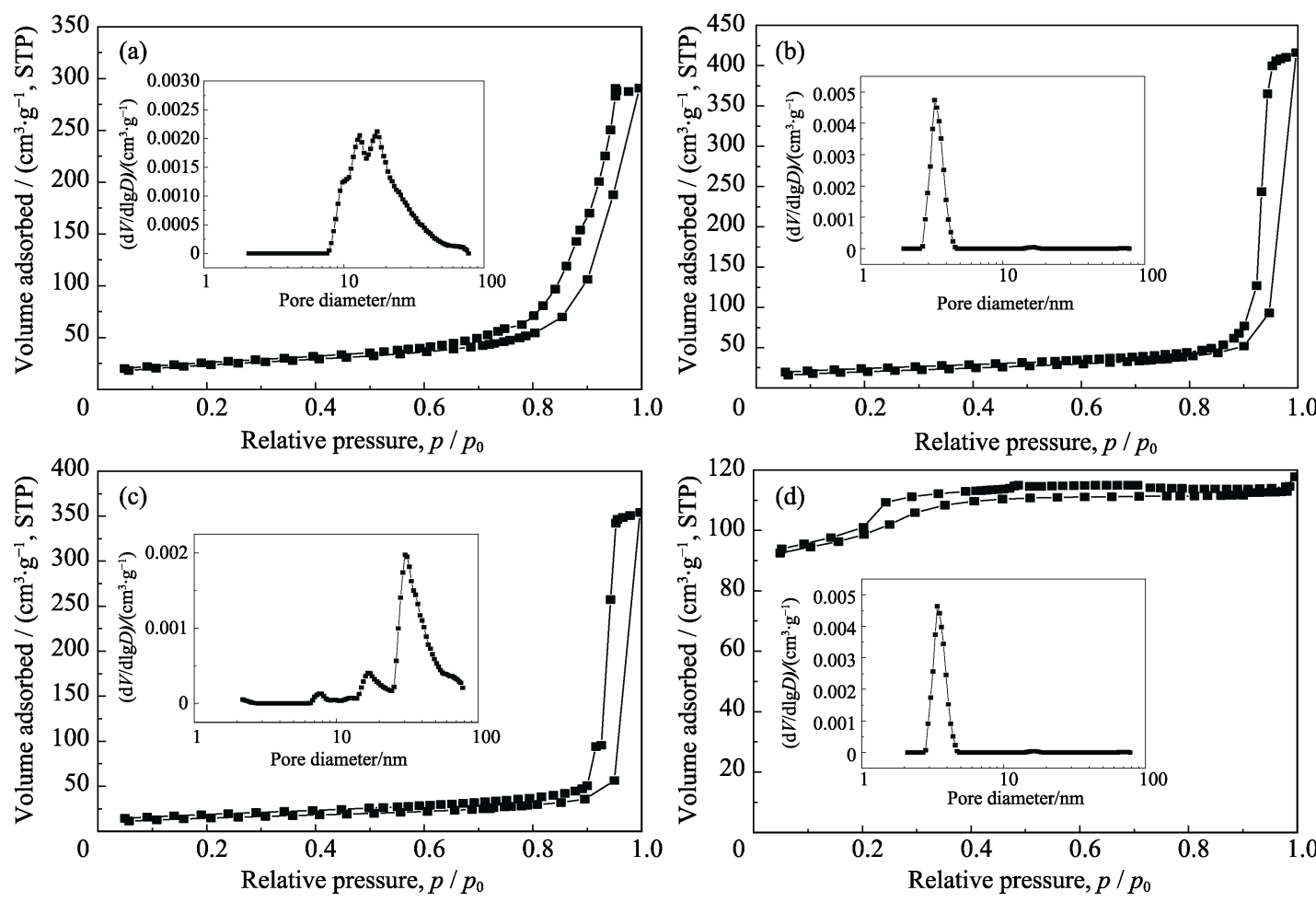

图 $3 \mathrm{Al}-\mathrm{SiO}_{2}$ 样品的 $\mathrm{N}_{2}$ 吸附一脱附曲线及孔径分布图

Fig. $3 \mathrm{~N}_{2}$ adsorption-desorption curves and pore size distributions of Si-Al samples (A) $\mathrm{Al}-\mathrm{SiO}_{2}(\mathrm{t}-80)$; (B) $\mathrm{Al}-\mathrm{SiO}_{2}(100)$; (C) $\mathrm{Al}-\mathrm{SiO}_{2}(120)$; (D) $\mathrm{Al}-\mathrm{SiO}_{2}(140)$

表 1 样品的孔结构参数

Table 1 Pore structure parameters of samples

\begin{tabular}{cccc}
\hline Sample & $S_{\mathrm{BET}} /\left(\mathrm{m}^{2} \cdot \mathrm{g}^{-1}\right)$ & $V_{\text {pore }} /\left(\mathrm{cm}^{3} \cdot \mathrm{g}^{-1}\right)$ & $D^{* / \mathrm{nm}}$ \\
\hline $\mathrm{Al}_{-} \mathrm{SiO}_{2}(\mathrm{t}-80)$ & 79.1 & 0.45 & 22.8 \\
$\mathrm{Al}_{-} \mathrm{SiO}_{2}(100)$ & 67.1 & 0.65 & 38.5 \\
$\mathrm{Al}_{-}-\mathrm{SiO}_{2}(120)$ & 49.1 & 0.55 & 44.5 \\
$\mathrm{Al}_{-} \mathrm{SiO}_{2}(140)$ & 304.7 & 0.18 & 2.4 \\
\hline
\end{tabular}

*: Average pore size $=4\left(V_{\text {pore }} / S_{\mathrm{BET}}\right)$

力较大的范围内伴有 $\mathrm{H} 2$ 型回滞环 ${ }^{[14,16]}$, 说明可能 存在由较大颗粒堆积而成的粒子间隙孔，介孔分布 较为宽泛; 而经过 $140{ }^{\circ} \mathrm{C}$ 处理的样品介孔回滞环不 明显, 孔径分布图显示样品在 2 3 $\mathrm{nm}$ 范围内有孔 分布, 说明存在一定量的表面介孔。 $\mathrm{N}_{2}$ 物理吸附表 征结果表明 $80{ }^{\circ} \mathrm{C}$ 水热处理获得的无定型 $\mathrm{Al}-\mathrm{SiO}_{2}$ 材料是双孔材料。结合 SEM 分析结果可知, 经过 $80 、 100 、 120{ }^{\circ} \mathrm{C}$ 水热处理得到的样品具有多级孔(大 孔一介孔)结构, 而通过 $140{ }^{\circ} \mathrm{C}$ 水热处理得到的样品 主要为微孔 ZSM-5 分子篮。从表 1 中孔结构参数亦 可知, 合成的多级孔材料具有较大的比表面积和 孔容。

\section{3 催化剂的表征}

催化剂的 XRD 图谱如图 4(A)所示, 多级孔 $\mathrm{Al}-\mathrm{SiO}_{2}(\mathrm{t}-80)$ 材料和商业 $\mathrm{SiO}_{2}$ 负载的钴催化剂均有 五个主要特征衍射峰, 分别位于 $2 \theta=31.6^{\circ} 、 36.8^{\circ} 、 44.9^{\circ}$ 、
59.6 $6^{\circ}$ 和 $65.4^{\circ}$, 对应 $\mathrm{Co}_{3} \mathrm{O}_{4}$ 的(220)、(311)、(400)、(511) 和 (440) 晶面的衍射峰 ${ }^{[17-18]}$ 。相比于 $\mathrm{Co} / \mathrm{SiO}_{2}$, $\mathrm{Co} / \mathrm{Al}-\mathrm{SiO}_{2}(\mathrm{t}-80)$ 的衍射峰较弱, 说明 $\mathrm{Co}_{3} \mathrm{O}_{4}$ 晶粒更 小。基于主峰 $2 \theta=36.8^{\circ}$ 处衍射峰强, 使用谢乐公式 计算了 $\mathrm{Co}_{3} \mathrm{O}_{4}$ 晶粒的大小, 可得商业 $\mathrm{SiO}_{2}$ 和多级孔 $\mathrm{Al}-\mathrm{SiO}_{2}$ 材料负载的钴催化剂中 $\mathrm{Co}_{3} \mathrm{O}_{4}$ 晶粒大小分 别为 9.8 和 $9.4 \mathrm{~nm}$ 。

催化剂的 TEM 照片如图 4(B)所示, 商业 $\mathrm{SiO}_{2}$ 中载体粒子较小, 紧密团聚形成介孔结构, 负载的 钴催化剂上 $\mathrm{Co}_{3} \mathrm{O}_{4}$ 颗粒聚在一起形成 50 100 $\mathrm{nm}$ 的 团簇; 相比于商业 $\mathrm{SiO}_{2}$ 负载的钴催化剂, 多级孔 $\mathrm{Al}-\mathrm{SiO}_{2}(\mathrm{t}-80)$ 材料负载的钴催化剂分散比较均匀, 催化剂中大颗粒载体堆在一起, 形成了较大的介孔 结构。结合 XRD 分析, $\mathrm{Al}-\mathrm{SiO}_{2}(\mathrm{t}-80)$ 材料负载的钴 催化剂的 $\mathrm{Co}_{3} \mathrm{O}_{4}$ 平均晶粒大小相近。多级孔结构 $\mathrm{Al}-\mathrm{SiO}_{2}(\mathrm{t}-80)$ 上 $\mathrm{Co}_{3} \mathrm{O}_{4}$ 团簇体分散度提高, 主要归因 于 $\mathrm{Al}$ 的掺杂和特殊的孔道结构防止了钴物种在催 化剂制备过程中发生团聚。

通过 $\mathrm{H}_{2}$-TPR 研究了催化剂的还原性。表征结 果如图 5(A)所示, 催化剂的两个主要还原峰分别属 于 $\mathrm{Co}_{3} \mathrm{O}_{4} \rightarrow \mathrm{CoO}(\alpha)$ 和 $\mathrm{CoO} \rightarrow \mathrm{Co}^{0}(\beta)$ 的两步还原, 商 业 $\mathrm{SiO}_{2}$ 载体负载的钴催化剂在 $450{ }^{\circ} \mathrm{C}$ 以上还存在 弱还原峰 $(\gamma)$, 这是由于钴物种与载体之间存在一定 的相互作用, 形成了难还原物种。从图 5(A)可以看 
出, $\mathrm{Co} / \mathrm{Al}-\mathrm{SiO}_{2}(\mathrm{t}-80)$ 相比 $\mathrm{Co} / \mathrm{SiO}_{2}, \alpha 、 \beta$ 还原峰温度 更高, 说明催化剂的还原性更低, 主要是由于 $\mathrm{Al}$ 掺 杂使得钴物种分散性显著提高, 钴物种和载体的相 互作用增强 ${ }^{[19-20]}$, 催化剂低温还原性降低。

\section{4 费托合成性能评价}

图 5(B)为催化剂的活性(CO 转化率) 随反应时 间的变化, 表 2 为催化剂的费-托合成催化性能评价

(B)
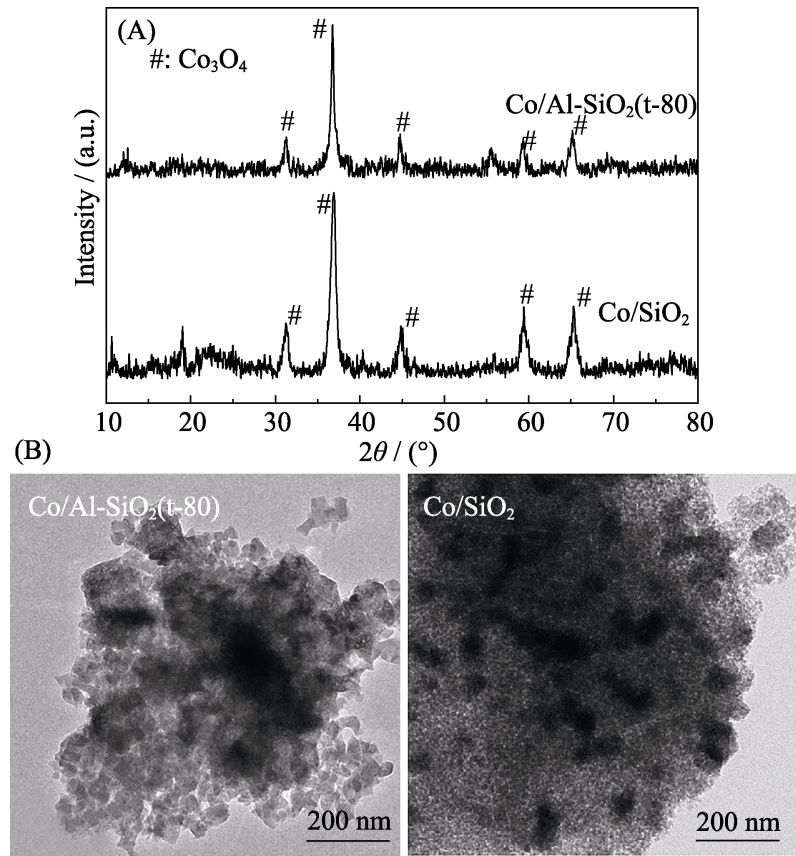

图 4 催化剂的 XRD 图谱(A)和 TEM 图(B)

Fig. 4 XRD patterns (A) and TEM images (B) of different catalysts
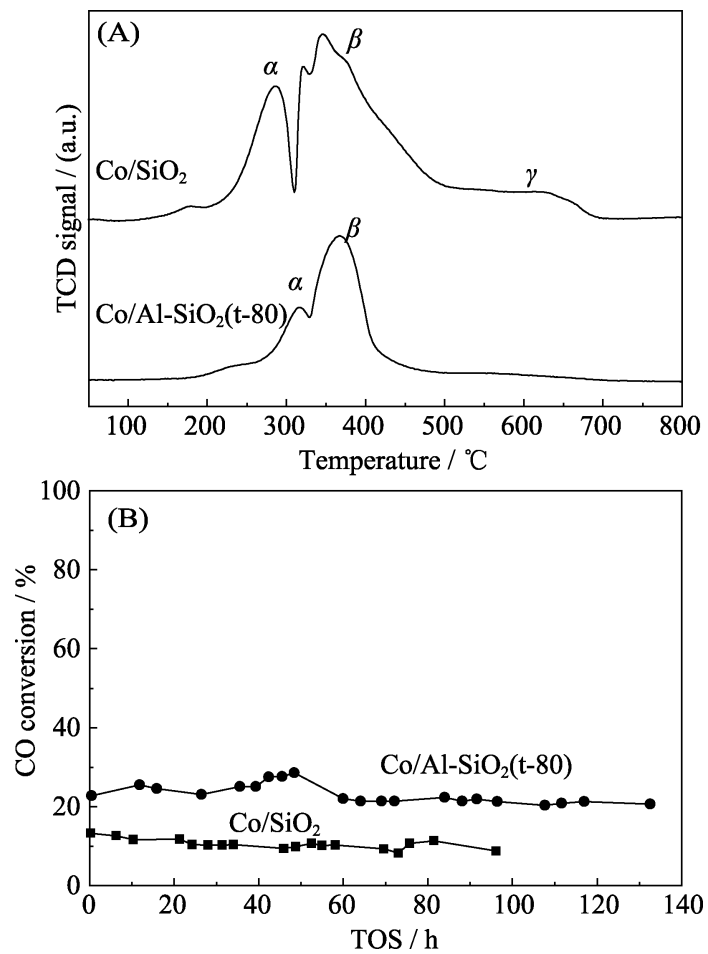

图 5 催化剂的 $\mathrm{H}_{2}-\mathrm{TPR}$ 图(A)和活性随时间的变化曲线(B) Fig. $5 \mathrm{H}_{2}$-TPR(A) and TOS(B) profiles of different catalyst
表 2 催化剂的费一托合成反应活性和选择性数据

Table 2 Activity and selectivity data of catalysts for Fischer-Tropsch synthesis

\begin{tabular}{ccccccc}
\hline \multirow{2}{*}{ Catalyst } & \multirow{2}{*}{ Load $/ \%{ }^{\mathrm{a}} \mathrm{CO}$ con. $/ \%$} & \multicolumn{4}{c}{$\mathrm{Sel} / \mathrm{wt} \%$} \\
\cline { 3 - 7 } & & & $\mathrm{CH}_{4}$ & $\mathrm{C}_{2}-\mathrm{C}_{4}$ & $\mathrm{C}_{5}-\mathrm{C}_{12}$ & $\mathrm{C}_{12+}$ \\
\hline $\mathrm{Co} / \mathrm{SiO}_{2}$ & 14.1 & 10.1 & 15.0 & 16.5 & 49.5 & 19.0 \\
$\mathrm{Co} / \mathrm{Al}-\mathrm{SiO}_{2}(\mathrm{t}-80)$ & 14.6 & 21.4 & 12.1 & 14.3 & 53.3 & 17.3 \\
\hline $\begin{array}{l}\text { Reaction condition: } 1.0 \mathrm{MPa}, 210 \\
{ }^{\mathrm{a}} \text { Measured by } \mathrm{C}, \mathrm{H}_{2} / \mathrm{CO}=2, \mathrm{GHSV}=4 \mathrm{~L} /(\mathrm{h} \cdot \mathrm{g})(>20\end{array}$ & $\left.{ }^{\circ} \mathrm{C}\right)$
\end{tabular}

结果, 从中可以看到, $\mathrm{Co} / \mathrm{Al}-\mathrm{SiO}_{2}(\mathrm{t}-80)$ 稳定后的平 均转化率为 $21.4 \%$, 是 $\mathrm{Co} / \mathrm{SiO}_{2}$ 的两倍。由表 2 中的 产物选择性数据可知, $\mathrm{Co} / \mathrm{Al}-\mathrm{SiO}_{2}(\mathrm{t}-80)$ 有较低的 $\mathrm{CH}_{4} 、 \mathrm{C}_{2}-\mathrm{C}_{4} 、 \mathrm{C}_{13}$ 选择性(12.1wt\%、14.3wt\%、17.3wt\%) 和较高的汽油段产物 $\left(\mathrm{C}_{5}-\mathrm{C}_{12}\right)$ 选择性 $(53.3 \mathrm{wt} \%)$ 。

综合催化剂表征结果可知, $\mathrm{Al}$ 元素掺杂显著提 高了多级孔 $\mathrm{Al}-\mathrm{SiO}_{2}(\mathrm{t}-80)$ 载体上钴物种的分散, 虽 然催化剂还原性有所降低, 但是相比于 $\mathrm{Co} / \mathrm{SiO}_{2}$ 催 化剂, $\mathrm{Co} / \mathrm{Al}-\mathrm{SiO}_{2}$ 催化剂能提供更多的活性中心，而 且多级孔(大孔一介孔)在反应过程中有利于反应物 和产物的传质扩散和二次反应的发生，降低了催化 剂低碳烃产物的选择性, 提高了 $\mathrm{C}_{5+}$ 的选择性 ${ }^{[5,9,21]}$ 。

\section{3 结论}

1) 研究表明水热法是合成多级孔 $\mathrm{Al}-\mathrm{SiO}_{2}$ 载体 材料的有效方法, 其中以 TEOS 为硅源, TPAOH 为 结构导向剂, 在 $80{ }^{\circ} \mathrm{C}$ 水热条件下可制得无定型介 孔-大孔 $\mathrm{Al}-\mathrm{SiO}_{2}$ 材料。

2) 利用等体积浸渍法将钴负载到多级孔 $\mathrm{Al}-\mathrm{SiO}_{2}$ 载体, 制备了钴基费-托合成催化剂, 催化 性能测试结果表明: 相比介孔载体, 多级孔结构的 形成提高了钴物种的分散度, 提高了 CO 转化率, 同时多级孔结构有利于反应物和产物的传质扩散, 降低了低碳烷烃选择性, 提高了汽油段 $\left(\mathrm{C}_{5}-\mathrm{C}_{12}\right)$ 产 物选择性，具有良好的应用前景。

\section{参考文献:}

[1] ADDIN EN REFLIST, XIONG H, ZHANG Y, et al. FischerTropsch synthesis: the effect of $\mathrm{Al}_{2} \mathrm{O}_{3}$ porosity on the performance of $\mathrm{Co} / \mathrm{Al}_{2} \mathrm{O}_{3}$ catalyst. Catalysis Communications, 2005, 6(8): 512-516.

[2] RAHMATI M, HUANG B, MORTENSEN M K, et al. Effect of different alumina supports on performance of cobalt FischerTropsch catalysts. Journal of Catalysis, 2018, 359: 92-100.

[3] LIU C, LI J, ZHANG Y, et al. Fischer-Tropsch synthesis over cobalt catalysts supported on nanostructured alumina with various morphologies. Journal of Molecular Catalysis A: Chemical, 2012, 363-364: 335-342.

[4] LI J, LI Q, ZHANG Y. Carbon-titania-composite supported cobalt catalyst for application in Fischer-Tropsch synthesis. Journal of 
South-Central University for Nationalities(Nat. Sci. Edition), 2013, 32(4): $1-6$.

[5] KOO H M, THANH T P, YI G R, et al. Effect of the ordered meso-macroporous structure of $\mathrm{Co} / \mathrm{SiO}_{2}$ on the enhanced activity of hydrogenation of $\mathrm{CO}$ to hydrocarbons. Catalysis Science \& Technology, 2016, 6(12): 4221-4231.

[6] XIONG H, ZHANG Y, LIEW K, et al. Fischer-Tropsch synthesis: the role of pore size for Co/SBA-15 catalysts. Journal of Molecular Catalysis A: Chemical, 2008, 295(1/2): 68-76.

[7] GEORGEW HUBER, STEVEN J M BUTALA, LEE M L, et al. Gd promotion of $\mathrm{Co} / \mathrm{SiO}_{2}$ Fischer-Tropsch synthesis catalysts. Catalysis Letters, 2001, 74(1/2): 45-48.

[8] DEN OTTER J H, NIJVELD S R, DE JONG K P. Synergistic promotion of $\mathrm{Co} / \mathrm{SiO}_{2}$ Fischer-Tropsch catalysts by niobia and platinum. ACS Catalysis, 2016, 6(3): 1616-1623.

[9] WEI L, ZHAO Y, ZHANG Y, et al. Fischer-Tropsch synthesis over a 3D foamed MCF silica support: toward a more open porous network of cobalt catalysts. Journal of Catalysis, 2016, 340: 205-218.

[10] LI H, HOU B, WANG J, et al. Effect of hierarchical mesomacroporous structures on the catalytic performance of silica supported cobalt catalysts for Fischer-Tropsch synthesis. Catalysis Science \& Technology, 2017, 7(17): 3812-3822.

[11] SONG D, LI J. Effect of catalyst pore size on the catalytic performance of silica supported cobalt Fischer-Tropsch catalysts. Journal of Molecular Catalysis A: Chemical, 2006, 247(1/2): 206-212.

[12] XIONG H, ZHANG Y, WANG S, et al. Preparation and catalytic activity for Fischer-Tropsch synthesis of Ru nanoparticles confined in the channels of mesoporous SBA-15. The Journal of Physical Chemistry C, 2008, 112(26): 9706-9709.
[13] CHEN M Q, WANG Y, YANG D Y, et al. Pt supported hierarchical ZSM-5 zeolite as adsorbent/catalytic combustion catalyst for o-xylene elimination. Journal of Inorganic Materials, 2019, 34(2): 173-178.

[14] SCHMIDT F, HOFFMANN C, GIORDANINO F, et al. Coke location in microporous and hierarchical ZSM-5 and the impact on the MTH reaction. Journal of Catalysis, 2013, 307: 238-245.

[15] YUE Y, KANG Y, BAI Y, et al. Seed-assisted, template-free synthesis of ZSM-5 zeolite from natural aluminosilicate minerals. Applied Clay Science, 2018, 158: 177-185.

[16] THOMMES M, SMARSLY B, GROENEWOLT M, et al. Adsorption hysteresis of nitrogen and argon in pore networks and characterization of novel micro- and mesoporous silicas. Langmuir, 2006, 22: 756-764.

[17] ZHAO Y, ZHANG Y, WEI L, et al. Effect of water on FischerTropsch synthesis over SBA-15 supported cobalt catalysts. SCIENTIA SINICA Chimica, 2014, 44(10): 1627-1632.

[18] GAO S, HONG J, XIAO G, et al. Evolution of cobalt species in glow discharge plasma prepared $\mathrm{CoRu} / \mathrm{SiO}_{2}$ catalysts with enhanced Fischer-Tropsch synthesis performance. Journal of Catalysis, 2019, 374: 246-256.

[19] ARNOLDY P, MOULIJN J A. Temperature-programmed reduction of $\mathrm{CoOAI}_{2} \mathrm{O}_{3}$ catalysts. Journal of Catalysis, 1985, 93(1): 38-54.

[20] STEEN E V, SEWELL G S, MAKHOTHE R A, et al. TPR study on the preparation of impregnated $\mathrm{Co} / \mathrm{SiO}_{2}$ catalysts. Journal of Catalysis, 1996, 162: 220-229.

[21] LUALDI M, DI CARLO G, L GDBERG S, et al. Effect of Ti and Al addition via direct synthesis to SBA-15 as support for cobalt based Fischer-Tropsch catalysts. Applied Catalysis A: General, 2012, 443-444: 76-86. 